\title{
RursuSpicae
}

Transmission, réception et réécriture de textes, de

I'Antiquité au Moyen Âge

1 | 2018

Parodies et pastiches antiques

\section{Wolf und Lamm. Ein Dialog über eine äsopische Fabel (= Phaedrus 1, 1)}

The Wolf and the Lam. Dialog on an Aesop's fable (= Phaedrus 1.1)

Le loup et l'agneau. Dialogue sur une fable ésopique

Cătălin Enache, Anna Balder, Anna Gsöllpointner et Hannah Müller

\section{(2) OpenEdition}

Journals

Édition électronique

URL : http://journals.openedition.org/rursuspicae/352

DOI : $10.4000 /$ rursuspicae.352

ISSN : 2557-8839

Éditeur :

Université Nice-Sophia Antipolis, IRHT - Institut de recherche et d'histoire des textes

Référence électronique

Cătălin Enache, Anna Balder, Anna Gsöllpointner et Hannah Müller, « Wolf und Lamm. Ein Dialog über eine äsopische Fabel (= Phaedrus 1, 1)», RursuSpicae [En ligne], 1 | 2018, mis en ligne le 15 octobre 2018, consulté le 27 avril 2021. URL : http://journals.openedition.org/rursuspicae/352 ; DOI : https:// doi.org/10.4000/rursuspicae.352

Ce document a été généré automatiquement le 27 avril 2021

RursuSpicae 


\title{
Wolf und Lamm. Ein Dialog über eine äsopische Fabel (= Phaedrus 1,1$)$
}

\author{
The Wolf and the Lam. Dialog on an Aesop's fable (= Phaedrus 1.1) \\ Le loup et l'agneau. Dialogue sur une fable ésopique
}

Cătălin Enache, Anna Balder, Anna Gsöllpointner et Hannah Müller

Das erste Gedicht in der Fabelsammlung des Phaedrus (1, 1) erzählt von einer Begegnung zwischen einem Wolf und einem Lamm. Bei dieser Begegnung findet ein Dialog zwischen den zwei Protagonisten statt. Der Erzähler, der die Szene beschreibt und den Dialog wiedergibt, ist kein neutraler Beobachter, sondern verfolgt eine eigene Strategie, die die Leser zu einer bestimmten Wahrnehmung des Erzählten führen soll, noch bevor die Moral ausgesprochen wird. Obwohl der Erzähler in der Szene nicht erscheint und am Dialog nicht teilnimmt, ist seine Präsenz unbestreitbar und seine Stimme unüberhörbar. Was er seinen Lesern allerdings nicht sagt, ist, dass er seinen eigenen Dialog mit Äsop führt, der vor ihm eine ähnliche Fabel erzählt hatte (155 in Perrys Sammlung). Ohne den äsopischen Text einfach ins Lateinische zu übersetzen, hält sich Phaedrus sehr nah an sein griechisches Vorbild. Ohne an der Handlung etwas $\mathrm{zu}$ ändern, bringt er eine neue Version auf die Bühne - wie ein Regisseur, der einer altbekannten Geschichte seinen Stempel aufdrückt (dasselbe wird dann auch Babrius in seiner Fabel 89 tun). Der Vielschichtigkeit und Vielstimmigkeit des Phaedrus-Gedichts vom Wolf und vom Lamm ist der folgende Dialog gewidmet. Die Autoren haben sich vorgenommen, die Begegnung zwischen den zwei Tieren aus vier verschiedenen Perspektiven zu betrachten und gemeinsam auszuloten, was diese Szene zu einer Fabel macht. Dabei wollten sie nicht so sehr endgültige Antworten geben, als vielmehr die zahlreichen leicht übersehbaren Aspekte beleuchten, die für das Verständnis dieser und jeder Fabel von Bedeutung sind.

\footnotetext{
Ad rivum eundem lupus et agnus venerant, siti compulsi. Superior stabat lupus, 
"Qui possum, quaeso, facere quod quereris, lupe?

A te decurrit ad meos haustus liquor."

Repulsus ille veritatis viribus

"Ante hos sex menses male" ait "dixisti mihi."

Respondit agnus "Equidem natus non eram."

"Pater hercle tuus" ille inquit "male dixit mihi"

atque ita correptum lacerat iniusta nece.

Haec propter illos scripta est homines fabula,

qui fictis causis innocentes opprimunt.

\section{Prolegomena zu einer Mahlzeit}

CĂTĂLIN ENACHE: Das ist ein einfacher Text, eine ganz einfache Szene. Zwei Tiere kommen zusammen, reden miteinander, dann frisst das eine das andere. Es wird letztendlich eine Mahlzeit oder die Vorbereitung einer Mahlzeit beschrieben. Kann man es so sehen? Seid ihr mit dieser Sicht einverstanden? Es ist ein Text, in dem das erzählt wird, was unmittelbar vor einer Mahlzeit stattfindet. Die Mahlzeit an sich ist ja ganz banal, nicht wahr? Wir essen alle, die Tiere essen auch alle. Die Frage wäre, auf der einen Seite, ob ihr mit dieser Beschreibung einverstanden seid, und auf der anderen Seite, warum diese Mahlzeit in einem Text erzählt zu werden verdient. Was ist so besonders dabei?

HANNAH MÜLLER: Ganz oberflächlich gesehen würde ich sagen: ja, es geht um die Vorbereitung einer Mahlzeit. Aber der Fokus liegt dabei nicht so wirklich auf der Mahlzeit selbst, sondern eher auf der Zubereitung der Mahlzeit, nämlich anhand der Konfrontation, die eben der Wolf beginnt. Er startet diese Konfrontation, diesen Streit, damit er das Lamm essen kann. Er will es so zu sagen nicht roh essen, also ohne Grund töten, sondern braucht einen Grund dafür, damit er sich nicht so schlecht fühlt. Deshalb sucht er zuerst einen Streit, damit er es rechtfertigen kann, dass er diese Mahlzeit isst.

CE: Wie man z.B. einen Apfel nicht direkt vom Baum isst, sondern inn zubereitet, indem man ihn schneidet und daraus einen Apfelstrudel macht? Ist das der Sinn des Textes?

HM: Wenn man die Fabel in einem Satz zusammenfassen muss und die Frage ist, ob es um die Vor- oder Zubereitung der Mahlzeit geht, dann würde ich sagen: vielleicht, oberflächlich, aber eigentlich geht es um die Gewissensberuhigung des Wolfes. Die zwei Aspekte hängen schon zusammen, aber es sind zwei verschiedene Ebenen.

CE: Was passiert im Text? Was erzählt unser Text?

ANNA GSÖLLPOINTNER: Ein Wolf trifft während des Trinkens an einem Fluss auf ein Lamm. Weil er dieses aber nicht ohne Grund töten möchte, sucht er einen Streit, um es töten zu können. Zuerst wirft er ihm vor, es verschmutze das Wasser. Diesen Vorwurf wehrt das Lamm ab, wie auch den folgenden Vorwurf, dass es ihn vor sechs Monaten beschimpft habe. Zuletzt wird es aber vom Wolf trotzdem getötet.

CE: Das heißt, zwei Tiere kommen zusammen.

AG: Ja.

CE: Das eine frisst das andere.

AG: Ja.

CE: Warum ist das eine Fabel?

AG: Diese Tiere stehen für archetypische Eigenschaften. Weil Tiere in Fabeln zumeist menschliche Eigenschaften verkörpern, sollen sie dadurch den Menschen einen 
Spiegel vorhalten. Deshalb sind ja diese Tiere auch nur Metaphern oder Symbole für menschliches Verhalten.

HM: Das habe ich mit den zwei Ebenen gemeint: Man kann lesen, dass ein Wolf ein Lamm frisst, aber man kann es auch auf einer tieferen oder moralischen Ebene sehen und herauslesen, was die Tiere für Eigenschaften verkörpern oder was diese Eigenschaften bedeuten sollen. So kann man dann die Moral der Fabel erkennen.

CE: Das heißt, wenn ich diesen Text lese, soll ich an zwei Menschen denken, von denen der eine den anderen frisst. Meint ihr das so?

HM: Nein.

AG: Nein.

CE: Versuchen wir das, was im Text erzählt wird, wie eine Bildgeschichte zu sehen. Zwei Tiere kommen zusammen. Das ist das erste Bild. Im zweiten Bild frisst das eine das andere. Warum ist das eine Fabel?

HM: Weil hier eben mehr passiert als nur, dass das eine das andere frisst.

CE: Das heißt, wenn ich den Text so zusammenfasse, habe ich das Wesentliche der Fabel ausgelassen.

HM: Ja.

CE: Was wäre das?

HM: Das ist genau was ich mit meiner Zusammenfassung gemeint habe, nämlich, dass der Wolf, um sein Gewissen zu beruhigen, dass er etwas Schlechtes begeht, Ausreden und Vorwände sucht, um das Lamm fressen zu können.

AG: Es wäre eben keine Fabel, wenn man nur sagen würde: Ein Wolf frisst ein Lamm. Die Szene wird genau dadurch zu einer Fabel, dass diese Tiere zum einen menschliche Eigenschaften verkörpern und zum anderen auch miteinander kommunizieren können. Das ist eben die Konfrontation. Durch diese Konfrontation soll dem Leser eine Nachricht mitgegeben werden, eine Lebensweisheit wird in diesem Falle durch einen Dialog zwischen zwei Tieren vermittelt.

HM: Ich würde es auch so sagen: Ein Lehrsatz, der in einer anderen Geschichte verpackt ist. Oder in einer Situation, die auf Tiere übertragen wird.

AG: Es ist eine Lebensweisheit, die anhand eines pointierten Beispiels bildnerisch oder spielerisch, eben durch die Tiere, dargestellt wird.

CE: Ihr geht jetzt davon aus, dass der Text selbstverständlich einen tieferen, weiteren Inhalt hat, der jedem Leser unmittelbar zugänglich ist. Versuchen wir den Text mit den Augen eines Kindes zu lesen. Denken wir an die Bildgeschichte. Im ersten Bild kommen die Tiere zusammen, wir könnten noch ein paar Bilder dazwischen geben, wo die Tiere miteinander reden, und im letzten Bild frisst das eine das andere. Wir wollen einem Kind erklären, dass das eine Fabel ist. Was würden wir sagen? Wir würden sagen, dass diese Tiere menschliche Züge haben. Was wären diese Züge? Haben nicht alle Tiere menschliche Züge? Haben Tiere überhaupt menschliche Züge? Was wären sie in unserer Geschichte? Du hast gesagt, dass sie miteinander reden.

AG: Und es ist am Schluss auch eine Moral zu finden, was ein wesentlicher Aspekt einer Fabel ist. Diese könnte man auch einem Kind erklären, oder zumindest 
näherbringen. Vermutlich wird auch das Kind einsehen: Das ist nicht in Ordnung, das ist böse, was der Wolf macht.

CE: Eine Moral muss nicht in jeder Fabel dabei sein, sie gehört auch nicht unmittelbar zum Text. Die Szene, die in einer Fabel beschrieben wird, muss einen Sinn ergeben, auch wenn der Fabelerzähler sie dem Leser nicht erklärt. Außerdem muss die Erklärung, die uns der Erzähler gibt, nicht die einzige oder die beste oder die richtige sein. Was der Erzähler bietet, ist nur eine mögliche Erklärung der Szene, genau wie jeder andere Betrachter oder Leser. In unserem Fall würde ich von der Moral des Phaedrus absehen. Sehen wir von den letzten zwei Versen einfach ab. Was bleibt übrig? Zwei Tiere kommen zusammen, sie haben menschliche Züge.

AG: Ja.

CE: Außer dass sie miteinander reden, was sind diese menschlichen Züge? Woran erkennt man sie? Ein Kind kann sich mit einem Tier immer identifizieren, z.B. mit einem Seehund im Tiergarten. Ein Kind freut sich, wenn es Tiere sieht, weil es in ihnen menschliche Züge erkennt. Das Tier bewegt sich, ist lebendig, man will mit ihm spielen, man sieht es gerne, man identifiziert sich spontan mit ihm. Haben die Tiere in unserem Text etwas, was andere Tiere nicht haben, wenn sie einem Kind so menschlich vorkommen?

HM: Ich würde sagen, dass sie nicht nur menschliche Züge haben, sondern, wie Anna schon gesagt hat, sie verkörpern Stereotype und Archetype. Das heißt, sie beschränken sich auf bestimmte Eigenschaften.

CE: Archetype und Stereotype sind aber keine Menschen, das ist ein Abstraktionsniveau, das sehr weit vom Text entfernt ist. Ich würde gerne wissen, woran ich erkennen soll, dass die Tiere in unserem Text für Menschen stehen. Bedenkt noch einmal die Frage, die ich früher gestellt habe: Soll man an zwei Menschen denken, von denen der eine den anderen frisst? Inwiefern geht es um Menschen in unserem Text?

AG: Wir müssen an das denken, was man aus dieser Konfrontation lernen soll und wie diese Konfrontation von statten geht. Das ist es, was man auf den Menschen ummünzen kann. Das Ende ist natürlich nicht so gewöhnlich bei einer Konfrontation zwischen Menschen. Aber dass der Wolf eine Art von Gewissen zeigt, das sieht man daran, dass er das Lamm nicht ohne Grund töten möchte.

CE: Woraus kann man schließen, dass der Wolf ein Gewissen hat?

AG: Er könnte es auch einfach so töten, ohne es anzusprechen. Aber er tut das, er spricht das Lamm an und sucht Streit, weil er Hunger hat. Das ist, was im Text steht.

CE: Das heißt, dass das Menschliche daran ist, dass er das Essen nicht direkt isst: die Zubereitung.

HM: Das Menschliche ist das Gewissen, und das Gewissen erkennt man daran, dass er das Essen nicht gleich isst, obwohl er es könnte - weil er offensichtlich stärker ist als das Lamm -, sondern noch irgendwelche Vorwände braucht.

AG: Um sein Gewissen zu befriedigen.

HM: Denn physisch hat der Wolf ja keine Schwierigkeiten, das Lamm umzubringen. Psychisch aber schon. Er hat eine Blockade, die sein Gewissen ist.

ANNA BALDER: Ich würde die menschlichen Eigenschaften, die da zu erkennen sind, viel direkter aus dem Text nehmen. Allein schon durch die Wörter, die der Erzähler verwendet. Z.B.: Warum sucht der Wolf Streit? Aus böser Fressgier, fauce improba. Alleine dieses Gierige und dieses Böse ist etwas, was ein Tier von Natur aus nicht hat. Ein Wolf sucht sich Beute, wenn er Hunger hat oder befürchten muss, dass er in nächster Zeit nichts mehr findet, und nicht, weil er jetzt gerade Lust hat, das kleine 
Lämmchen zu töten. Und diese Intention, die dahinter steckt, die auch durch den Erzähler und seine Wortwahl vermittelt wird, ist ein Hinweis darauf, dass der Wolf mehr ist, als nur ein Wolf, dem man vielleicht im Wald begegnen würde. Und das geht noch weiter, denn der Wolf versucht, sich ziemlich ungewöhnlich zu rechtfertigen: Warum soll ich das Lamm noch fressen, wenn es gar nicht das Wasser trüben kann, oder wenn es nicht das Lamm ist, das mich beleidigt hat?! Diese Rechtfertigungen würde ein Tier auch nicht suchen. Die Rechtfertigung für einen normalen Wolf, wenn er im Wald Hunger hat, ist: Ich habe Hunger und jetzt esse ich so viel ich brauche, und nicht: Ich habe Hunger und töte, weil ich gerade Lust darauf habe, oder weil ich mich für eine Beleidigung räche, oder weil mir das Lamm etwas angetan hat. Das ist eher eine Art von menschlicher Eigenschaft, die impliziert, dass der Wolf eben etwas rechtfertigen muss. Und rechtfertigen muss er, dass er das Lamm tötet.

CE: Soll ich schließen aus dem, was du sagst, dass die Menschen, bevor sie essen, sich rechtfertigen, wieso sie essen müssen?

AB: Nein, nein. Ich würde dieses Fressen eher metaphorisch betrachten, denn es geht nicht um das Fressen von anderen Menschen. Dieses Fressen bedeutet vielmehr jemand anderem Leid zufügen oder jemand anderen vernichten oder einfach durch eigene Handlungen Schlechtes zufügen. Dann sucht man irgendeinen Grund, oder eher einen Vorwand, der die Rolle hat, die eigene Handlung zu rechtfertigen, auch wenn er so nicht wirklich zutrifft.

CE: Das heißt, wenn ich dich recht verstehe, der Text ist deiner Meinung nach gar nicht über eine Mahlzeit.

\section{Ungerechtigkeit und Macht}

ANNA BALDER: Ja, ich würde weg von der Mahlzeit gehen, wenn man ihn wirklich tiefer betrachten möchte. Wenn wir auf der Tierebene bleiben, dann kann man über Fressen durchaus diskutieren. Aber wenn wir jetzt von menschlichen Eigenschaften reden, dann ist die Interpretation Der Text ist über eine Mahlzeit nicht wirklich zutreffend, denn das Fressen ist metaphorisch anzusehen.

HANNAH MÜLLER: Ich würde sagen, dass die Tierebene da ist, um das Ganze irgendwie zu verpacken. Man sagt es eben nicht direkt. Es gibt ja viele Fabeln und generell Geschichten, die auch Kritik üben - aber irgendwie verpackt, um nicht Leute direkt zu attackieren, die Ähnlichkeiten mit den kritisierten Figuren hätten, und auch um es leichter verständlich zu machen. Die Tierebene ist da, um das zu verpacken.

CĂTĂLIN ENACHE: Wenn wir also die Szene auf die Menschen übertragen, dann entspricht dem Essen am Ende das Leidzufügen.

AB: Ja. Oder dem anderen schaden. Oder, wie der Erzähler im letzten Vers sagt, unterdrücken, opprimere. Das ist auch nicht zufällig das letzte Wort des Textes. Aber ich glaube, dass es gar nicht so weit gehen muss. Es reicht schon, wenn man durch ungerechtfertigte Handlungen bewirkt oder ungerechte Handlungen dazu führen, dass ein anderer Nachteile hat.

CE: Das heißt, der Text ist aus deiner Sicht über...

AB: Ungerechtigkeit von verschiedenen mächtigen Personen. Über diesen Machtunterschied, der zwischen Lamm und Wolf herrscht. Der Wolf ist ein Raubtier, hat große Zähne, ist kräftig, stark, schnell, kann weit springen. Das Lamm ist ein 
Huftier, ein Nutztier für uns Menschen, eigentlich schon domestiziert und außerdem noch dazu ein Lamm, also ein kleines Schaf, im Gegensatz zum ausgewachsenen Wolf. Das ist ein großer Macht- und Kraftunterschied. Und ich würde dieses Verhältnis so einbeziehen, dass man metaphorisch sagt, der mit der größeren Kraft schadet dem Schwächeren.

CE: Ich glaube auch, dass das Machtverhältnis ein wesentlicher Aspekt dieser Szene ist, den wir bis jetzt nicht klar genug hervorgehoben haben. Es sind ja nicht irgendwelche zwei Tiere, die da zusammenkommen, sondern ein stärkeres und ein schwächeres. Was mir allerdings nicht ganz klar ist, ist, warum wir glauben sollen, dass der Text eigentlich die Gerechtigkeit zum Thema hat. Das Machtverhältnis ist auf jeden Fall klar. Aber Gerechtigkeit? Was soll der Wolf tun, wenn er Hunger hat? Warum ist das eine Ungerechtigkeit, wenn er doch nur sein Bedürfnis stillt? Warum soll der Inhalt des Textes die Gerechtigkeit oder die Ungerechtigkeit sein, wenn der Wolf den anderen nicht tötet, um seine Macht zu zeigen, oder um ihn zu unterdrücken, oder um ihn zu unterwerfen, sondern nur um sein natürliches Hungergefühl zu stillen?

AB: Aber der Wolf hat kein natürliches Hungergefühl. Der Erzähler sagt, dass er zum Fluss gekommen ist, weil er Durst hatte, siti compulsus. Auch steht im Text ausdrücklich, dass er das Lamm "aus böser Fressgier" angreift, fauce improba, das heißt mit einer bösartigen Intention. Hunger zu haben ist ja nichts Böses, jeder hat mal Hunger, jeder muss essen, um zu überleben, das ist verständlich.

CE: Wenn man also aus dem Text fauce improba streicht, dann enthält die Szene keine Ungerechtigkeit mehr?

AB: Nein, weil der Wolf dann ja mit den Rechtfertigungen noch weiter kommt: "Du hast mich beleidigt", "Ach nein, dein Vater hat mich beleidigt, deswegen töte ich dich".

ANNA GSÖLLPOINTNER: Außerdem bezeichnet der Erzähler selbst die Tötung als ungerecht, denn im Vers 13 sagt er lacerat iniusta nece.

AB: Eigentlich wollte ich gar nicht sagen, dass Gerechtigkeit der Hauptansatzpunkt ist, den man bei der Lektüre nehmen sollte. Ich habe Gerechtigkeit nur deswegen eingebracht, weil der Wolf versucht, sich zu rechtfertigen - sich selbst gegenüber oder eventuell auch anderen gegenüber, das kann ich jetzt nicht beurteilen. Es könnte durchaus auch sein, dass der Wolf seinen Ruf aufrecht erhalten will, wenn wir das wirklich auf Menschen beziehen. Seine Argumentation wäre dann: "Ich töte niemanden aus irgendeinem Grund, sondern ich habe einen richtigen Grund gehabt, daher bin ich trotzdem immer noch genau so edel wie zuvor, weil ich aus einem guten Grund getötet habe." An sich ist aber egal, welchen Grund er nennt, z.B. Rache für eine fiktive Beleidigung. Die Gerechtigkeit kommt aber erst dann ins Spiel, wenn man noch einen Schritt weiter macht und sich fragt, wie der Wolf bei der Tötung des Lammes vorgeht. Natürlich, einfach so zu töten, ohne Grund, ist ohnehin ungerecht. Aber weil er das auch noch zu kaschieren versucht, macht er alles noch schlimmer.

CE: Versuchen wir noch einmal, uns die Szene neutral anzuschauen, ohne uns von Hilfestellungen des Erzählers wie fauce improba oder iniusta nece verleiten zu lassen. Ein Wolf trifft auf ein Lamm. Er hat wahrscheinlich Hunger, oder ist zumindest nicht satt. Er diskutiert mit dem Lamm, genauer gesagt: er macht ihm Vorwürfe. Das Lamm versucht sich zu verteidigen, letztendlich kommt es zum naheliegenden Schluss, dass der Mächtigere den Schwächeren tötet und frisst. Was ist hier das Ungerechte? Wenn ich nicht irre, ist der Begriff Gerechtigkeit in unserem Gespräch dann aufgetaucht, als wir gesagt haben, dass es dem Wolf klar ist, dass er das Lamm nicht einfach so töten kann. Wir haben aus den Vorwürfen des Wolfes einen Rechtfertigungsversuch geschlossen und dann die 
Rechtfertigung als Beweis dafür betrachtet, dass er selbst die Tötung als ungerecht empfindet. Mir ist allerdings diese Verbindung zwischen Rechtfertigung und Ungerechtigkeit nicht ganz klar. Der Wolf will nur etwas essen. Was ist die Ungerechtigkeit?

AG: Die Ungerechtigkeit besteht für mich darin, dass das Lamm nie eine Chance gehabt hat, egal was es tut. Es hat objektiv keine Chance zu entkommen oder zu überleben, nachdem es mit diesem Wolf zusammengetroffen ist. Es hat keine Überlebenschance und das ist die Ungerechtigkeit.

CE: Dann liegt aber die Ungerechtigkeit nicht an den Rechtfertigungen des Wolfes. Denn das Lamm hat von Anfang an keine Chance, auch wenn der Wolf sich nicht rechtfertigen würde.

AG: Aber genau das ist es, dass der Wolf es nicht einfach so tötet, sondern eine Konfrontation sucht und ihm dabei eine scheinbare Chance gibt. Die Vorwürfe des Wolfes sind offensichtlich ungerecht, weil er das Lamm weiter und weiter beschuldigt, obwohl es alle Vorwürfe entkräftet. Am Schluss tötet er es einfach nur mehr aus Ungeduld, denn das letzte rechtfertigt er gar nicht mehr. Er sagt nur noch “beim Herkules!" und tötet das Lamm.

CE: Aber worin besteht die Ungerechtigkeit? Darin, dass der Wolf dem Lamm überlegen ist? Oder darin, dass der Wolf das Lamm tötet? Oder darin, dass der Wolf dem Lamm Vorwürfe macht? Oder darin, dass der Wolf die Antworten des Lammes nicht gelten lässt? Oder darin, dass der Wolf dazu gezwungen ist sich von anderen Tieren zu ernähren? Worin besteht die Ungerechtigkeit?

AG: Für mich besteht sie darin, wie er mit dem Lamm und mit dessen Antworten während der Konversation umgeht. Also darin, dass er keine dieser logischen Antworten gelten lässt und immer persönlicher wird. Das, was er vorwirft, hat letztendlich nichts mehr mit logischen Gründen und Rechtfertigung zu tun.

CE: Ob man das aber als Ungerechtigkeit bezeichnen kann? Ich glaube, dass man das Prädikat ungerecht in erster Linie für Taten verwendet: Jemand hat etwas Ungerechtes getan, seine Tat war ungerecht. Worte oder Gedanken können vielleicht auch als ungerecht betrachtet werden, aber genau genommen ist die Handlung ungerecht. Die Ungerechtigkeit zeigt sich hauptsächlich in Handlungen, nicht in Worten oder Gedanken.

HM: Meiner Meinung nach ist die Tötung das Ungerechte. Nicht weil auf der Tierebene ein Wolf ein Lamm tötet, sondern, wie wir die Szene vorher metaphorisch betrachtet haben, weil der stärkere dem schwächeren Leid zufügt. Das ist das Ungerechte.

AB: Nehmen wir an, der Wolf hätte keine böse Absicht, er hat einfach Hunger und will das Lamm fressen. In diesem Fall wäre der Dialog gar nicht nötig. Das Lamm bemerkt den Wolf, rennt weg, der Wolf hinterher. Der Wolf ist stärker, schneller, größer, erwischt das Lamm und kann seinen Hunger stillen. Die Szene würde genau so gut widerspiegeln, wie sehr der Wolf dem Lamm von Anfang an überlegen ist, egal was das Lamm macht.

CE: Die Frage ist nun: Was bringt der Dialog dazu? Denn die Szene ist so, wie du sie beschreibst, wenn man den Dialog auslässt.

HM: Ich würde noch einmal sagen, dass die Ungerechtigkeit die Tötung des Lammes ist bzw. die Tatsache, dass dem Schwächeren Leid zugefügt wird. 
CE: Wir müssen aber nicht vergessen, dass die Tötung nicht den Sinn hat, die eigene Überlegenheit zu zeigen.

HM: Nein, sie soll das eigene Bedürfnis befriedigen. Die Fressgier, die wir weggestrichen haben, bezeichnet in Wirklichkeit den eigenen Vorteil und ist genauso metaphorisch zu betrachten. Wenn die Tötung nicht eine wirkliche Tötung, sondern das Leidzufügen symbolisiert, dann steht die Fressgier nicht direkt für den Verzehr, sondern für den Vorteil, den man aus einer Handlung zieht. Was der Dialog für mich hinzufügt, ist das Gewissen. Aus dem Dialog kann man herauslesen, dass der Wolf, dem das Leid des Lammes einen Vorteil bringt, ein schlechtes Gewissen darüber hat.

AG: Kann man den Dialog der zwei Tiere nicht auch als einen Prozess des Quälens verstehen?

CE: Zubereitung?

AG: Ja, Zubereitung. Das ist ein absichtliches Quälen, weil das Lamm keine Chance hat, egal was es tut.

CE: Ein sadistischer Wolf, also.

AG: Genau. Er sucht gezielt diese Konversation, obwohl er sehr wohl weiß, dass er das Lamm töten wird, egal was dieses tut und antwortet. Er hat vielleicht zu sich selbst gesagt: "Ich töte es, egal was es antwortet, aber vorher spiele ich noch ein bisschen mit ihm." Auch eine Katze, die eine Maus töten will, spielt zuerst noch ein bisschen mit ihr und lässt sie ein bisschen leiden, anstatt sie gleich zu töten.

CE: Ob die Katze Gewissensfragen hat, wenn sie mit der Maus herumspielt, bevor sie sie tötet?

AG: Die Katze erfreut sich am Leid. Ob man von einem Gewissen reden kann, weiß ich nicht, vielleicht gibt es irgendeine Fabel, in der auch eine Katze Gewissensfragen hat. Ich glaube aber, dass der Wolf durch diesen Dialog das Lamm quält, und die Fabel zeigt, dass der Unterlegene nicht nur Unrecht erleidet, sondern auch ungerechterweise gequält wird.

CE: Das wäre aber gerade das Menschliche dabei, nicht wahr? Seinen Nächsten quälen, das kann doch der Mensch besser als jedes andere Tier. Darin kennt sein Erfindungsgeist keine Grenzen, kein Tier kann mit ihm mithalten. Wenn wir uns fragen, was in dieser Szene auf die Menschen übertragen werden kann, dann müssen wir auch das Katz-und-Maus-Spiel nennen, das eine verbreitete Beschäftigung vieler Menschen ist. Was die Katze mit der Maus macht ist nur ein Spiel im Vergleich zum Katz-und-Maus-Spiel der Menschen.

HM: So etwas kann man allerdings wirklich bei Katzen beobachten.

CE: Sie werden es bei den Menschen gelernt haben.

AG: Meine Katze hat einmal eine Maus hochgebracht: Sie blutete schon, aber die Katze hat sie noch durch die Wohnung rennen lassen. Die Maus hat herumgequiekt und irgendwann, als sie komplett am Ende war, hat die Katze sie getötet. Sie hat sie aber nicht gefressen, sondern wieder vor die Tür gelegt.

CE: Hast du das als ungerecht empfunden?

AG: Ich war klein und habe es eher als eklig empfunden. Im Nachhinein empfinde ich es aber schon als Quälerei, vor allem weil die Katze die Maus auch nie gefressen hat: Sie hat sie verletzt, gequält und hat dabei Freude darüber empfunden, wie das Tier gelitten hat. So würde ich auch das Verhalten des Wolfes in dieser Fabel sehen, es ist ein Quälen und Töten aus Spaß. 
CE: Wenn es aber so ist, dann können wir diesen Aspekt nicht mehr als menschliches Verhalten einstufen. Wenn Tiere so etwas natürlich machen, dann ist das Quälen des Lammes durch den Wolf nicht mehr das Menschliche an dieser Szene. Aber ich glaube nicht, dass das viele Tiere machen.

AG: Das Quälen kommt hier durch die Konversation heraus, und das ist eindeutig eine menschliche Eigenschaft.

CE: Wo bleibt dann die Ungerechtigkeit?

AG: Das Quälen ist das Ungerechte.

CE: Der Wolf quält also das Lamm mithilfe der Sprache und handelt dabei ungerecht, das heißt, die Ungerechtigkeit zeigt sich im sprachlichen Verhalten des Wolfes. Die Tatsache, dass er mit dem Lamm noch verhandelt, wäre dann das Ungerechte.

AG: Weil der Ausgang ihm und jedem anderen von Anfang an klar ist. Würde der Wolf das Lamm nur töten, dann wäre es einfach ein biologischer Prozess. Er möchte vorher aber noch seinen Spaß daran haben und dabei vielleicht auch noch sein Gewissen bereinigen. Denn das Lamm versucht alle Vorwürfe zurückzuweisen und schafft es auch, aber das ist dem Wolf egal. Er quält es aus Spaß, dann reißt sein Geduldsfaden und er tötet es, weil er auf der Argumentationsebene nicht mehr weiter weiß. Im Text steht, dass das Lamm “ängstlich” entgegnet, timens. Daraus liest man wieder das Machtverhältnis zwischen den zwei Tieren. In Wirklichkeit ist aber das Lamm dem Wolf intellektuell überlegen, denn es gewinnt offensichtlich die verbale Auseinandersetzung. Das Lamm ist schlagfertig, kann sich gut verteidigen, hat immer die beste Antwort parat, der Erzähler sagt auch, dass die Wahrheit auf seiner Seite ist, veritatis vires. Das kann ein Mächtigerer natürlich nicht ertragen.

AB: Eigentlich steht am Ende nicht explizit, dass er es frisst. Ich glaube, dass dieser Aspekt, dass er es tötet, um es zu fressen, nicht ausdrücklich erwähnt ist. Der Erzähler sagt zum Schluss lacerat, also der Wolf zerfleischt das Lamm. Zerfleischen heißt aber nicht unbedingt fressen. Wenn man die nackte Naturszene nimmt "Wolf trifft auf Lamm, jagt es, erwischt es und zerfleischt es", dann denkt man freilich spontan an Fressen. Aber der Dialog zwischen den zwei Tieren rückt diesen Aspekt "Hunger" und "Fressen" ein bisschen in den Hintergrund, finde ich, weil es jetzt hauptsächlich darum geht, dass der Wolf einen Grund oder einen Vorwand sucht. Außerdem steht dann am Ende gar nicht deutlich fest, dass er es frisst. Und wenn er es nicht frisst, sondern nur für seine Frechheit bestraft, dann handelt er noch ungerechter, als wenn er es einfach so ohne Grund getötet und dann gefressen hätte.

CE: Ich glaube, dass man das Fressen schon mithören kann, auch wenn wir fauce improba auslassen. Eigentlich ist improba das Störende, denn faux ist ein neutraler Hinweis auf den Hunger oder auf das Fressen.

HM: Ich glaube, dass es eigentlich egal ist, ob er es am Ende frisst oder nicht.

AB: Der Dialog ist de facto der wichtigste Teil, der die Möglichkeit zum Interpretieren öffnet. Ohne Dialog wäre alles sehr einfach. Die Szene wäre auch keine Fabel mehr. In Wirklichkeit besteht die Fabel in diesem Dialog, die Rahmenszene ist nur wegen des Dialogs da. Die Vorwürfe des Wolfes und die Erwiderungen, die Widerlegungen des Lammes machen den Konflikt aus, und die Interpretation wird dadurch emöglicht, dass sich der Konflikt im Dialog abspielt. Aufgrund der Wahrheit und der Tatsachen, dass das Lamm den Fluss nicht trüb gemacht haben konnte und noch nicht geboren war, als der Wolf gemeint hat, dass es ihn beleidigt habe, ist es ungerecht, dass der Wolf es tötet. Der Wolf tötet es aus Gier und vielleicht dafür, dass er genervt ist vom 
Lamm, oder dafür, dass er sich in seiner Machtstellung angegriffen fühlt, weil das Lamm klug ist und auf die Vorwürfe erwidern konnte. Das ist dann die Ungerechtigkeit.

AG: Der Wolf sucht absichtlich einen Grund, um es töten zu können. Er findet aber irgendwie keinen und so entsteht eine Ungerechtigkeit. Denn er erweckt den Anschein, dass er einen Grund braucht, um es zu töten, tötet es dann aber doch, auch wenn er keinen Grund gefunden hat. Darin besteht für mich die Ungerechtigkeit, und auch darin, dass er es durch den Dialog quält.

HM: Wäre es gerechter, wenn er einen Grund hätte? Denn der Erzähler sagt zum Schluss, dass der Wolf das Lamm "ungerechterweise tötet", lacerat iniusta nece. Aber können wir uns eine gerechte Tötung vorstellen? Kann er wirklich einen guten Grund für seine Handlung vorbringen, so dass wir dann sagen können, dass er das Lamm zu Recht getötet hat? Z.B. er trifft auf ein Lamm, das ihn tatsächlich beleidigt hat, hat außerdem Hunger und denkt sich: "Ah, wie gut, dass ich auf dieses Lamm treffe, das ist doch gerade jenes Lamm, das mich damals beleidigt hat, jetzt kann ich es töten!" Wäre es dann gerechtfertigt? Nehmen wir an, dass der Wolf keine leeren Vorwürfe macht, sondern das Lamm ihm tatsächlich etwas angetan hatte. Geht dann die Tötung in Ordnung?

AG: Er bräuchte in Wirklichkeit keinen Grund, um es zu fressen.

HM: Natürlich, aber wenn wir meinen, dass die Ungerechtigkeit darin besteht, dass er keinen Grund findet...

AG: Das liegt daran, dass er einen Grund finden will und den Anschein erweckt, dass er einen braucht, um das Lamm zu töten.

HM: Genau, und er tötet es dann trotzdem, auch wenn er keinen gefunden hat, weil er "böse" und "ungerecht" ist. Aber wenn er einen Grund hätte, wäre dann seine Handlung, d.h die Tötung, gerecht?

AG: Sie würde zumindest seinen Voraussetzungen entsprechen, d.h. er würde konsequent handeln.

HM: Also er könnte noch mehr sein Gewissen beruhigen?

AG: Ja.

CE: In so einem Fall würden wir von Rache für ein erlittenes Unrecht sprechen. Und die Frage wäre dann, ob die Rache, d.h. die Strafe, in einem angemessenen Verhältnis zum erlittenen Unrecht steht. Ansonsten kommt Tötung als Strafe nicht nur in Fabeln vor, und ob das jeweils erlittene Unrecht diese Strafe rechtfertigt, ist auch nicht immer klar. Es gibt auf jeden Fall Gesetzgebungen, die die Todesstrafe vorsehen. Sie wird dann als gerecht betrachtet werden müssen, wenn das geltende Gesetz das Kriterium der Gerechtigkeit ist. Der eigentliche Unterschied zu unserer Situation wäre aber, dass wir in diesem Fall keine Fabel mehr hätten. Mich interessiert in erster Linie, was diese Szene und diesen Text zu einer Fabel macht, was das Fabel-hafte an der Fabel ist, und erst dann die anderen Aspekte, die für das Verständnis der konkreten Situation von Bedeutung sind, wie Gerechtigkeit und Ungerechtigkeit. Denn das Fabel-hafte muss schon im Text enthalten sein, sonst würde es nicht jedem Leser spontan einleuchten, heute wie vor zweitausend Jahren, dass er mit einer Fabel zu tun hat, während die anderen Aspekte mehr oder weniger im Auge des Betrachters liegen. Übrigens könnten wir uns auch fragen, was passieren würde, wenn das Lamm einfach nichts mehr zu erwidern hätte, z.B. wenn der Wolf eine Frage stellen würde, die das Lamm nicht beantworten könnte, etwas in der Art der Sphinx. Wenn die Rettung 
des Lammes von der Beantwortung der Frage abhängen würde und das Lamm wirklich die Antwort nicht wissen würde, wäre dann die Handlung, also die Tötung gerecht?

HM: Oder wenn das Lamm nicht so intelligent wäre und die konkrete Situation einfach nicht begreifen würde. Es bemerkt z.B. nicht, dass der Fluss in die andere Richtung fließt und kann dem Vorwurf nichts entgegnen. Denn in unserem Text stellt der Wolf keine Fragen, sondern beschuldigt direkt das Lamm. Er fragt nicht: "Hast du mich beleidigt?", sondern sagt: "He, du hast mich beleidigt!", und das Lamm entgegnet, dass er ihn nicht beleidigt haben kann.

CE: Stimmt, aber er könnte auch Fragen stellen. Denn die Vorwürfe zielen darauf ab, eine "Schuld" beim Anderen festzustellen, die dann die Strafe rechtfertigt. Der Wolf könnte aber auch anders versuchen, die "Schuld" festzustellen, nämlich indem er das Lamm in irgendeiner Form auf die Probe stellt, also prüft: "Kannst du die Antwort nicht, dann bist du tot." Eine Kapitalprüfung, so zu sagen, wo die Prüfungsfrage alles entscheidet. Der Wolf würde dann auf dasselbe Ergebnis kommen, wie wenn er Vorwürfe macht: Er würde "die Schuld nachweisen."

AG: Aber woher nimmt er sich das Recht, so eine Kapitalfrage zu stellen? Wie kann es spontan dazu kommen, dass der eine der Prüfer und der andere der Geprüfte ist?

AB: Wenn ich richtig verstehe, geht es jetzt um die Frage, was Gerechtigkeit innerhalb eines festgelegten Rahmens bedeutet. Wenn die Bedingungen durch eine Art Vertrag von Anfang an definiert sind, dann hat man bei der Interpretation der Gerechtigkeit natürlich nicht mehr so viel Spielraum.

AG: Aber dass man sich als Überlegener das Recht nehmen darf, andere zu töten, wenn man ihnen eine "Schuld nachweisen" kann? Warum soll man da von Recht reden? Ist nicht jeder und alles gleich?

CE: Also in der Natur ist der eine stärker und der andere schwächer, und manchmal ernährt sich der Stärkere vom Schwächeren. Die klassische Staatstheorie behauptet, dass der Naturzustand durch den Gesellschaftsvertrag überwunden wird. Das ist eine Sammlung von Vorschriften und Verboten, die das Zusammenleben regeln sollen und für alle verpflichtend sind, die Teil der Gesellschaft sein wollen. Jeder, der glaubt, dass das Leben in der Gemeinschaft vorteilhafter ist als der Naturzustand, muss sich daran halten. Unser Text ist diesbezüglich zweideutig, denn man kann nicht sofort sagen, ob man einen Gesellschaftsvertrag voraussetzen darf oder nicht. Einerseits findet die Szene in der Natur statt und die Darsteller sind Tiere, so dass man als Beobachter geneigt sein kann, das Recht des Stärkeren gelten zu lassen. Andererseits sieht jeder Leser spontan ein, dass die Tiere nur Rollen sind und dass es in Wirklichkeit um Menschen geht, so dass man sich genauso gut auf den Gesellschaftsvertrag berufen kann, der ja den Mord verbietet. Einerseits geht es um Nahrung und Hunger, der der natürlichste aller Triebe ist und eher an den tierischen Zustand denken lässt, andererseits reden die Tiere und ihr Gespräch ist ein wesentlicher Aspekt der Szene, so dass man wiederum an menschliches Zusammenleben erinnert ist.

AG: Wenn ein hungriger Wolf ein Lamm frisst, dann hat die Szene keine weitere Bedeutung. Unser Wolf quält aber das Lamm, indem er mit ihm diskutiert und ihm falsche Vorwürfe macht.

AB: So kommt auch das Gewissen des Wolfes deutlicher zum Vorschein, anders als wenn er wirklich nur Hunger hat. Er könnte zu sich sagen: "Ich habe Hunger, ich darf das Lamm fressen." Er denkt sich aber: "Ich versuche etwas zu finden, damit das Lamm es mir nicht zu übel nimmt, oder die anderen, oder ich es mir selbst nicht zu übel nehme." Denn das ist ungefähr der Sinn des Dialogs, er dient der Gewissensberuhigung des Wolfes. 
HM: Man erkennt auch, dass er es vor sich selbst rechtfertigt, weil es niemand anderen gibt, der ihn verurteilen kann. Nicht einmal das Lamm, denn das Lamm wird getötet. Er rechtfertigt seine Handlung vor sich selbst.

\section{Tier und Mensch}

CĂTĂLIN ENACHE: Wir könnten uns auch die folgende Frage stellen: Warum ist man als Leser geneigt, sich eher mit dem Lamm zu identifizieren als mit dem Wolf? Wir essen ja alle, oft auch Tiere, Tiere fressen auch, viele davon fressen andere Tiere. Dass heißt, Tiere werden ständig gefressen, sei es von Menschen oder von anderen Tieren. Warum sind wir aber als Zeugen dieser Szene spontan geneigt, uns eher mit dem Gefressenen als mit dem Fressenden zu identifizieren? Denn dass man von Ungerechtigkeit vor allem dann spricht, wenn man sie erleidet, ist klar. Wenn man Unrechtes tut, stört es nicht, oft bemerkt man es gar nicht. Wenn wir also diesen Aspekt beiseite legen und annehmen, dass es dem Wolf in erster Linie um Nahrung geht, dass er also das Lamm tötet, um sich ernähren zu können, warum lädt die Szene eher zum Nachdenken ein, anstatt z.B. unseren Appetit zu erregen?

HANNAH MÜLLER: Ich glaube, dass der Leser ab dem Zeitpunkt Mitleid empfindet, wo er das Opfer vermenschlicht bzw. wo er begreift, dass das Lamm für etwas anderes steht, nämlich für Menschen. Das gilt dann unabhängig von der Art der Tiere, die im Text vorkommen. Wenn die Geschichte nur von Wölfen handeln würde und nebenbei erwähnt werden würde, dass einer von den Wölfen ein Lamm gefressen hat, dann ginge es um irgendein Lamm, zu dem man nichts Näheres weiß. In unserer Fabel steht aber das Lamm im Mittelpunkt, es kann denken und fühlen, und weil es personifiziert ist, empfinden wir Mitleid, egal ob der Wolf ebenfalls vermenschlicht ist oder nicht. Das Entscheidende ist, wie der Erzähler die Szene beschreibt: In unserem Fall hält er eindeutig zum Lamm, das erkennt man einerseits an jenen Ausdrücken, die wir ausklammern mussten, um die Szene neutral zu beobachten, nämlich fauce improba oder iniusta nece, andererseits aber auch daran, dass der Fokus auf dem Lamm liegt.

CE: Das Lamm in unserer Geschichte ist also individualisiert - es ist dieses eine Lamm, das zum falschen Zeitpunkt am falschen Ort ist, das auf Vorwürfe eingehen kann, das Eltern hat - sein Vater wird im Text genannt. Auch wissen wir, dass er Angst hat (laniger timens), und das ist ein Gefühl, das jeder Mensch kennt. So ein allgemeines Gefühl verbindet den lesenden Menschen mit dem dargestellten Tier mehr als alles andere. Es ist nicht nur die Tatsache, dass das Lamm reden kann, denn das können in Fabeln alle Tiere, sondern vielmehr die konkrete Situation, die malerisch beschrieben wird: ein Fluss, am Ufer ein Lamm, weiter oben ein Wolf - er ist ohnehin der Überlegene, kein Zufall, dass er weiter oben steht -, sie haben Durst, sie reden miteinander, sie scheinen sich zu kennen, oder sie lernen zumindest im Laufe des Textes einander kennen. Man fragt sich fast, wieso das Lamm, das doch in einer Herde zu leben pflegt, sich von den anderen Schafen entfernt hat und alleine zum Fluss gegangen ist. Das Essen des Wolfes ist also kein herumlaufendes Stück Fleisch, vielmehr muss man sagen, dass ein konkretes Lamm, das man auch als Leser bei der Lektüre allmählich kennenlernt, ein Individuum, das vernünftige Antworten gibt und nachvollziehbar reagiert, am Ende gefressen wird. Das ist ja keine zufällige Momentaufnahme aus dem Naturalltag, sondern eine bestimmte Situation, in der ein Darsteller zum Opfer wird.

HM: Zu dem Zeitpunkt, ab dem das Lamm nicht mehr ein Lamm, sondern das Lamm ist, hat man sich mit ihm schon identifiziert. Man kennt es schon, man fühlt mit ihm mit. Der Handlungsträger ist dann das Lamm. Daher ist es durchaus von 
entscheidender Bedeutung, ob am Ende irgendein Lamm oder dieses eine Lamm getötet wird.

ANNA GSÖLLPOINTNER: Was das Lamm in dieser Situation fühlt, ist nicht schwer nachzuvollziehen. Wir brauchen nur an eine Auseinandersetzung mit einer Autoritätsperson denken. Autoritätspersonen sind auch stark und mächtig, wie der Wolf. Sie sitzen am längeren Hebel. Man kann selten etwas gegen sie tun. Egal was man tut oder sagt, man hat trotzdem nicht die Macht, man kann nicht die Oberhand bekommen. Und das ist auch eine Ungerechtigkeit des Lebens, dass der Stärkere die Macht hat, auch wenn er im Unrecht ist.

CE: Macht bedeutet nicht nur über mehr Mittel als die anderen Menschen zu verfügen, sondern auch in geringerem Maß Konsequenzen zu befürchten. Ich glaube, dass wir noch einen Begriff in Zusammenhang mit dem Machtverhältnis in dieser Fabel nennen müssen, nämlich Willkür. Hier geht es nicht nur um Macht, sondern um willkürliche Machtausübung. Allerdings kann Willkür auch eine Sache der Perspektive sein. Machtpersonen treffen Entscheidungen, die viele Menschen betreffen: Wenn du als Betroffener dich nur auf dich selbst konzentrierst und die Gesamtperspektive außer Acht lässt, kann die Entscheidung einer Machtperson, die dir einen Nachteil gebracht hat, dir auch willkürlich erscheinen.

AG: Ich glaube, dass der Fabelerzähler sehr wohl beabsichtigt hat, dieses Gefühl der Machtlosigkeit im Leser hervorzurufen. Der Wolf ist im Recht, weil er die Macht hat, zu bestimmen, wer recht hat. Dass der Stärkere und Mächtigere tun und lassen kann, was er will, ist, glaube ich, auch eine Lebensweisheit, die der Fabelerzähler vermitteln will.

CE: Es gibt schon in der antiken Literatur die Idee, dass Gerechtigkeit das ist, was der Stärkere als solche definiert, und dass Ungerechtigkeit das Machtlosigkeitsgefühl des Schwächeren ist, der in Wirklichkeit genau dasselbe tun würde wie der Stärkere, wenn er die Möglichkeit hätte und keine Strafe zu befürchten hätte. Die Anfechter des Naturrechts, von denen einige in Platons Dialogen zu Wort kommen, behaupteten, dass in der Natur jeder tut und tun darf, was er kann, und dass der Begriff Gerechtigkeit nur in Zusammenhang mit Menschengemeinschaften einen Sinn hat. Die Gesetze, so heißt es, wurden als Schutz erfunden, nämlich von schwachen Menschen, die einen Schutz vor den Übergriffen der Stärkeren auch brauchen. Im Naturzustand setzt sich der Stärkere von selbst durch, daher braucht ein starker oder mächtiger Mensch den Schutz durch Gesetze auch nicht, weil er sich vor Übergriffen selbst verteidigen kann. Über Ungerechtigkeiten beklagt sich also nur ein Schwacher. Ähnliche Ideen sind auch bei Nietzsche zu finden, der z.B. in der Genealogie der Moral sophistisches Gedankengut ohne weiteres übernommen hat. Die Frage wäre nun: bringen wir vielleicht das Thema Ungerechtigkeit in den Text hinein, weil wir uns mit dem Lamm identifizieren, und nur dann, wenn wir uns mit ihm identifizieren? Oder ist es eher umgekehrt, wir identifizieren uns mit dem Lamm, weil wir das Gefühl haben, dass es ungerecht behandelt wird? Gibt es eigentlich die Möglichkeit, dass Leser sich nicht spontan mit dem Lamm identifizieren, sondern die Szene entweder neutral wahrnehmen oder sich gar mit dem Wolf identifizieren? Was würden dann solche Leser vom Thema Gerechtigkeit halten? Oder sollen wir eher glauben, dass jemand, der sich nicht spontan mit dem Lamm identifiziert, das deshalb tut, weil er schon eine Auffassung von Gerechtigkeit hat, die in Richtung Naturrecht geht?

AB: Ich denke schon, dass es auch Personen gibt, die sich nicht sofort oder überhaupt nicht mit dem Lamm identifizieren, aber meiner Meinung nach wird der Großteil der Leser durchaus Situationen erlebt haben, in denen sie sich ungerecht behandelt gefühlt haben, daher werden sie die Szene mit den Augen des Lammes sehen.

CE: Das wäre nach den Sophisten die Ursache der Gesetzte: Es gibt viel mehr schwache Menschen als starke und die Nachteile, die man hat, wenn man ein Unrecht erleidet, sind viel größer als die Vorteile, die man hat, wenn man ein Unrecht zufügt. Somit einigen sich 
die Menschen - d.h. die schwachen, denn sie sind die Mehrheit, und die Mehrheit entscheidet -, sowohl auf das Unrechttun als auch auf das Unrechterleiden zu verzichten, und sanktionieren das durch Gesetze.

ANNA BALDER: Jedenfalls, dadurch, dass man bereits auf die eine oder andere Art Ungerechtigkeit erlebt hat oder sich selbst schwach und hilflos gefühlt hat, ist es leichter sich mit dem Lamm zu identifizieren. Es wird kaum jemanden geben, der trotz großer Macht oder einer hohen Stellung, nicht noch jemanden mächtigeren über sich hat. Denken wir z.B an einen Prinz, der auf seinen Hauslehrer hören muss. Der Prinz selbst hat eine hohe Stellung inne und viele Möglichkeiten, aber der Hauslehrer steht über ihm, weil er vom König mit der nötigen Autorität ausgestattet wurde. Und selbst wenn jemand die größte Macht haben sollte, muss er im Laufe seines Lebens mit mächtigeren Personen Kontakt gehabt haben, z.B. mit den Eltern. Das heißt, auch wenn die Person jetzt mächtig und stark ist, hat sie irgendwann, z.B. als Kind, sicherlich Situationen erlebt, in denen sie mit einer Autorität konfrontiert war und auch Ungerechtigkeit empfunden hat. Außerdem bleiben Situationen, in denen man ein Unrecht erlitten hat, besser in Erinnerung als Situationen, in denen man selbst ungerecht war, weil man nicht gerne wahrnimmt, wie man andere selbst schlecht behandelt. Es ist einfacher über Ungerechtigkeit zu klagen, als darüber nachzudenken, ob oder wann man selbst ungerecht war. Auch sind unangenehme Gefühlszustände schnell wieder ins Gedächtnis zu rufen, wenn man durch einen Text wie diesen förmlich mit der Nase auf eine vorherrschende Ungerechtigkeit gestoßen wird.

AG: Ob es aber nicht auch Machtpersonen gibt, die sich durch einen solchen Text in ihrem Handeln vielleicht sogar bekräftigt fühlen, wenn sie sehen, dass der Wolf im Grunde genommen bemüht war, den Anschein der Ehrlichkeit irgendwie zu bewahren? Denn das ist der Sinn der Unterredung, die der Tötung vorangeht. Zwingt uns das Leben in der Gesellschaft nicht immer wieder dazu, den Schein aufrechtzuerhalten, oft auch wenn alle Beteiligten wissen, dass die Wirklichkeit anders ist? Wer in einer Gesellschaft lebt, muss auf den Schein Wert legen, das wissen wir alle, und das könnte auch eine Lehre dieser Fabel sein. Wir spielen alle allzu oft den Wolf.

HM: Aber der Wolf wird in der Fabel als Lügner dargestellt, weil seine Vorwürfe so einfach zu widerlegen sind. Er kommt hier auf jeden Fall schlecht weg und du würdest dich nicht mit dem Schlechten identifizieren wollen. Selbst wenn du jemanden angreifst, würdest du nicht sagen: Ich bin wie der Wolf, ich behandle jeden schlecht und nutze jeden aus und ziehe auch noch Vorteile daraus. Außerdem bin ich ein Lügner, nicht nur den anderen, sondern auch mir selbst gegenüber, denn meine Rechtfertigungen glaube ich selber nicht.

AG: Aber er lügt, um ein Ziel zu erreichen. Die Lüge ist nur ein Instrument. Und wer kennt dieses Instrument nicht? Ich glaube, dass wir uns oft auch selbst lügen, z.B wenn wir uns im Nachhinein selbstloses Handeln zuschreiben, oder wenn wir einfach etwas verdrängen, was wir offensichtlich falsch gemacht haben oder wofür wir uns schämen. Es gibt auch Lügen, die einem guten Zweck dienen. Im Alltag ist es auch nicht immer leicht, einen klaren Unterschied zwischen Lüge und Wahrheit oder zwischen Schein und Wirklichkeit zu machen. Wir leben meistens in einer Grauzone, es kommt oft auf den Blickwinkel an. Es gibt genug Konflikte, wo beide Parteien recht haben. In dieser Fabel ist der Erzähler auf der Seite des Lammes, daher beschreibt er 
die Szene einseitig, aber die Realität ist komplexer. Man kann wie der Wolf handeln, ohne sich bewusst mit diesem Wolf zu identifizieren. Ich meine vor allem das Verhalten des Wolfes vor der Tötung, nicht die Tötung selbst. Wir geben oft Gründe an, an die wir selbst nicht glauben.

AB: Eine Person, die wie der Wolf handelt, braucht aber nicht unbedingt Gründe zu erfinden, wenn sie die eigenen Handlungen nicht als ungerecht empfindet. Sie versteht möglicherweise nicht, dass die eigenen Taten anderen Menschen ungerecht erscheinen. Der Wolf in unserer Fabel gesteht sich aber selbst ein, dass er ungerecht handelt, sonst würde er nicht versuchen, sich zu rechtfertigen. Er sieht schon ein, dass das Lamm ein schuldloses Opfer ist.

AG: Ich glaube, dass man den Wolf auch anders als durch die Augen des Fabelerzählers sehen kann. Wir handeln oft ungerecht, obwohl wir sehr gut wissen, dass das, was wir machen, nicht in Ordnung ist. Das kann unterschiedliche Gründe haben. Manchmal ist das Unrecht nur eine Kleinigkeit, manchmal hoffen wir, dass niemand unser Fehlverhalten bemerken wird, manchmal ist die Versuchung einfach zu groß und wir können uns nicht widersetzen, manchmal überwiegt der erhoffte Vorteil das eingegangene Risiko. Gerade in solchen Fällen ist man bereit, die eigene Handlung im Nachhinein nicht nur vor anderen, sondern auch vor sich selbst zu rechtfertigen.

AB: Aber auch dann muss der Wolf in der Fabel trotzdem nicht die bevorzugte Figur zur Identifikation sein.

HM: Sobald man eingesteht, dass der Wolf nicht böse war, hat ja bereits eine Identifikation mit dem Wolf stattgefunden.

AG: Ob man sich mit dem Wolf oder mit dem Lamm identifiziert, kann auch von deiner Erziehung oder den Wertvorstellungen der Gesellschaft, in der du lebst, abhängen. Dieser Text ist ziemlich alt und wurde in sehr verschiedenen Epochen gelesen. Wir können nicht wissen, was jeder Leser empfunden hat. Wir wissen auch nicht, wie Machtpersonen prinzipiell denken, man sagt ja, dass die Macht jeden ändert.

\section{Moral?}

CĂTĂLIN ENACHE: Ich schlage vor, das wir zu einer weiteren wichtigen Frage kommen. Aus jeder Fabel sollte man etwas lernen. Was soll man aus dieser lernen? An wen ist sie gerichtet, an Wölfe oder an Lämmer? Was soll man hier beherzigen? Wie soll ich mein Verhalten ändern, nachdem ich gelesen habe, wie der böse Wolf das arme Lamm getötet hat? Die Szene ist da: Der eine frisst, das andere wird gefressen. Was soll man daraus mitnehmen?

ANNA GSÖLLPOINTNER: Ist es nicht eine Art Lebens- bzw. Überlebensweisheit, die einem da mitgegeben wird? Denn moralisch zu hinterfragen ist dieser Text allemal. Er zeigt, dass es immer einen Stärkeren gibt, der die Macht hat und viel mehr Rechte hat, die er sich herausnimmt oder herausnehmen kann. So einer ist im Überlebenskampf stärker als du, auch wenn er mit seinem Handeln und Tun im Unrecht ist.

CE: Aber um diese Idee auszudrücken, ist ja keine Fabel notwendig. Wir haben gesagt, dass das Eigentliche dieser Fabel im mittleren Teil besteht, wo der Wolf mit dem Lamm redet. Das Machtverhältnis ist von Anfang an da, daher ist auch der Ausgang vorhersehbar. Das kann nicht die Lehre der Fabel sein. Wenn man Wolf und Lamm hört, dann hat man das 
Machtverhältnis schon vor den Augen. Ich glaube nicht, dass diese Szene einfach nur darauf abzielt, dieses Machtverhältnis zu bestätigen. Das Besondere bei diesem Treffen ist der Dialog und die Tatsache, dass der Wolf sich vor sich selbst zu rechtfertigen versucht. Was soll man daraus lernen, nachdem wir die Szene beobachtet haben? Wir sehen jetzt von der Moral des Fabelerzählers ab. Ich habe mich z.B. spontan mit dem Lamm identifiziert. Was soll ich in Zukunft anders machen?

AG: Die Fabel schenkt dem Leser nicht gerade viel Hoffnung.

\section{CE: Was lernt man?}

AG: Man lernt z.B., dass es gut und wichtig ist für gewisse Dinge wie die Wahrheit einzustehen, denn das ist, was das Lamm in seinem Überlebenskampf letztendlich tut. Der Text ist allerdings ziemlich entmutigend, weil das Lamm für seine Rechte steht und logische Gründe anführt, was aber am Schluss trotzdem nichts bringt. Daher kann man sich schon fragen: Wozu eigentlich etwas tun? Man stirbt sowieso.

CE: Aber was lernt man? Ich glaube nicht, dass der Erzähler die Szene deshalb erzählt hat, um Menschen dazu zu bewegen, auf diese Welt voller Wölfe zu verzichten oder Selbstmord zu begehen. Wenn ich mich mit dem Lamm identifiziere, sehe ich ein, dass ich keine Chance habe, sobald ich zum falschen Zeitpunkt am falschen Ort bin. Ist das alles?

ANNA BALDER: Es wird deutlich, dass mit Macht auch Willkür einhergehen kann. Das heißt, in einem bestehenden Machtverhältnis kann es zu willkürlichen Handlungen des Mächtigeren kommen. Das Machtverhältnis wird hier konkret dargestellt. Die Lehre ist also: Auch wenn man als einzelner Mensch oder Bürger nicht viel ausrichten kann, wenn ein Herrscher Willkür übt, ist noch nicht alles verloren, denn Macht ist nicht gleich Recht. Der Mächtigere macht, was er will, aber was gerecht und ungerecht ist, kann er nicht bestimmen. Ich glaube, dass der Text der Gerechtigkeitsauffassung der Sophisten widerspricht.

HANNAH MÜLLER: Wenn man sich mit dem Lamm identifiziert, kann man für sich herausnehmen, dass alle ein Gewissen haben, auch die Bösen.

CE: Das soll man lernen?

HM: Ich denke schon, dass man das mitnehmen kann - wenn man sich vor Augen hält, dass auch die, die mich unterdrücken, menschliche Eigenschaften haben.

CE: Vor allem, wenn sie Menschen sind.

HM: Vor allem dann, genau. Sie können auch Mitleid empfinden und haben ein Gewissen.

CE: Das heißt, für dich ist wichtig, dass man auch im schlimmsten Fall eine Chance hat in eigener Sache zu argumentieren. Das wäre ein positiver Aspekt, auch wenn er hier nicht viel bringt.

AB: Man kann sich durchaus ein Beispiel am Lamm nehmen und vielleicht nur etwas anders agieren. Denn das Lamm ist ja klug, es ist imstande, den Wolf zu widerlegen, und wenn man die eigene Klugheit überlegt einsetzt, könnte sich auch eine solche Situation anders entwickeln. Das Lamm wirkt auch etwas naiv. Obwohl es sich offenbar fürchtet, versucht es, den Wolf durch reine Argumente zu überzeugen, was diesen nur noch wütender macht. Aber wenn diese Naivität des jungen Lammes wegfällt und man sich seiner Situation bewusst wird, könnte man vielleicht die eigene Klugheit anwenden, um einer schwierigen Situation zu entrinnen. Das Lamm ist jedenfalls aufgrund seines Verstandes nicht völlig unbewaffnet in diesem Kampf mit dem Wolf, auch wenn das in diesem Fall nicht gereicht hat. 
CE: Das ist schon eine Extremsituation.

HM: Das hat dann mit dem Gewissen nicht mehr so viel zu tun.

AB: Aber das Lamm könnte mit seiner Klugheit durchaus erkennen, dass der Wolf zumindest eine Art von Gewissen hat, und sich somit dem Wolf gegenüber anders verhalten. Es könnte z.B. das Mitleid des Wolfes erregen, d.h. dessen Gewissen gegen ihn verwenden, um zu entkommen.

AG: Man könnte auch lernen, dass man sich das Leben vereinfachen kann, wenn man Machtpersonen nicht widerspricht. Geht man solchen Situationen aus dem Weg, hat man ein einfacheres Leben. Aber auch aus dem Verhalten des Wolfes kann man etwas lernen, egal ob man sich mit ihm identifiziert oder nicht. Denn hier sieht man, wie man etwas bekommt, was man will aber nicht haben darf: Du schnappst es dir. Und wenn du nachher kein schlechtes Gewissen haben willst, bringst du Scheingründe vor.

HM: Jemand, der so wie der Wolf handelt, könnte aber auch lernen, dass dünne Vorwände nicht reichen, um wahre Motive zu verstecken, dass man also früher oder später enttarnt werden kann.

CE: In diesem Fall müssen wir dem Fabelerzähler dankbar sein, der mit seinem Text den Wolf bloßgestellt hat. Ohne ihn hätten wir nicht gewusst, dass ein böser Wolf einen Mord begangen hat und dass ein armes Lamm ohne Schuld gestorben ist. Er war dabei, hat alles beobachtet und der Nachwelt diesen schlagenden Beweis hinterlassen. Die Literatur als Zeugnis vergangener Untaten. Wer den Text kennt, weiß, was damals passiert ist und was wieder passieren kann.

AG: Die Literatur zeigt menschliche Verhaltensweisen auf, und diese sind nicht immer ideal und perfekt und gerecht. Diese Tiere haben ein menschliches Verhalten, daher müssen wir für sie Verständnis haben.

\section{RÉSUMÉS}

Le dialogue qui suit, entre les quatre auteurs, porte sur la fable „le Loup et l'Agneau“ (1.1), reprise d'Esope. Plusieurs questions sont abordées dans la discussion comme : „en quoi ce poème est-il une fable?", „quel est le véritable thème du poème“, „que veut communiquer l'auteur?“, "que peut-on apprendre de cette scène?“, "quel rôle joue le dialogue dans cette altercation?", „quel est l'enjeu de ce conflit?“, „dans quelles situations un semblable conflit peut-il surgir?“.

Der vorliegende Dialog zwischen den vier Autoren behandelt Phaedrus' Fabel über den Wolf und das Lamm $(1,1)$. Im Gespräch wird Fragen nachgegangen wie „Warum ist dieses Gedicht eine Fabel?", „Was ist das Thema des Gedichts?“, „Was wollte der Autor vermitteln?“, „Was soll man als Leser lernen?“, „Welche Rolle spielt der Dialog in der Auseinandersetzung?“, „Worin besteht der Konflikt?“, „In welchen Situationen kann ein ähnlicher Konflikt vorkommen?“.

The present dialogue deals with Phaedrus' fable about the wolf and the lamb $(1,1)$. In this discussion the four authors address questions like "Why is this poem a fable?", "What is the subject matter of the poem?", "What did the author want to tell us?", "What should we learn 
from the scene?", "What should we make of the fact that the two protagonists talk with one another?", "What are the main features of the confrontation?", "In which situations might a similar confrontation occur?".

\section{INDEX}

Keywords : Aesopus, Phaedrus, Fable, Wolf, Lamb, Natural Rights, Justice Mots-clés : Esope, Phèdre, Fable, loup, agneau, droit naturel, justice

Schlüsselwörter : Äsop, Phaedrus, Fabel, Wolf, Lamm, Naturrecht, Gerechtigkeit

\section{AUTEURS}

\section{CĂTĂLIN ENACHE}

hat Philosophie und Klassische Philologie in Bukarest studiert und ist Doktor in Philosophie der Universität Wien. Er hat die Didaktik der alten Sprachen an der Universität Wien gelehrt und Aufsätze zur antiken Philosophie und Medizin veröffentlicht. Er unterrichtet Latein und Altgriechisch in Wien. Anna Balder, Anna Gsöllpointner und Hannah Müller waren zum Zeitpunkt des Dialogs über Phaedrus‘ Fabel Schülerinnen.

\section{ANNA BALDER}

War Schülerin im Wiedner Gymnasium, Wien

\section{ANNA GSÖLLPOINTNER}

War Schülerin im Wiedner Gymnasium, Wien

\section{HANNAH MÜLLER}

War Schülerin im Wiedner Gymnasium, Wien 